\title{
A Note on variational solutions to SPDE perturbed by Gaussian noise in a general class
}

\author{
Michael Röckner* \\ Department of Mathematics and BiBoS, Bielefeld University, Bielefeld, Germany; \\ Email: roeckner@math.uni-bielefeld.de \\ Yi Wang * \\ Department of Mathematics, Purdue University, West Lafayette, USA;
}

September 2, 2021

\begin{abstract}
This note deals with existence and uniqueness of (variational) solutions to the following type of stochastic partial differential equations on a Hilbert space $\mathcal{H}$

$$
d X(t)=A(t, X(t)) d t+B(t, X(t)) d W(t)+h(t) d G(t)
$$

where $A$ and $B$ are random nonlinear operators satisfying monotonicity conditions and $G$ is an infinite dimensional Gaussian process adapted to the same filtration as the cylindrical Wiener pocess $W(t), t \geq 0$.
\end{abstract}

\section{Introduction}

Recently there has been an enormous interest in the study of Hilbert space-valued SPDE with fractional Brownian motion (fBm) representing the perturbing noise. The first paper on this topic we are aware of is the work by W.Grecksch and V.V. Anh. [5], followed by many others (see e.g. [2], [3], 4], 7], [9, [8], [11] and the references therein). The main thrust of the research efforts is now to allow the coefficient in the integral with respect to $\mathrm{fBm}$ to depend on the solution. But since Itô stochastic integration does not apply (because $\mathrm{fBm}$ is not a semimartingale), one has to use Skorohod stochastic integration or a pathwise integration approach. Both require more restrictive conditions on the coefficients of the equation. The purpose of this paper is another direction, namely sticking with additive noise we generalize the assumptions on the coefficients. In particular, we want to include

${ }^{*}$ This work has been supported by NSF-Grant 0603742 
nonlinear equations which are not semilinear in order to include more general classical PDE perturbed by Gaussian noise. We do this on the basis of [5] (i.e. we use the well-known technique of substracting the additive noise), but allow much more general drift coefficients and even start with an SPDE with a standard Wiener type noise which we disturb by another Gaussian (non-semimartingale) type noise. More precisely, our equation looks as follows

$$
d X(t)=A(t, X(t)) d t+B(t, X(t)) d W(t)+h(t) d G(t)
$$

with $A$ and $B$ being random and satisfying standard monotonicity conditions (cf. (H1)-(H4) below), $W(t), t \geq 0$, a cylindrical Wiener process and $G(t), t \geq 0$, an infinite dimensional Gaussian process with suitable integrand $h$ to be specified below.

One main contribution in this note is to specify a class of $G$ and $h$ as above so that one can use results from the variational approach to solve SDE (1.1) by the said technique of subtracting the additional noise

$$
\int_{0}^{\cdot} h(t) d G(t)
$$

Another contribution is to provide new examples of SPDE to which our results apply.

Below we only summarize the main results and sketch the ideas of the proofs. Detailed proofs and additional results are contained in [12]

\section{A class of Gaussian integrals}

Let $(\Omega, \mathcal{F}, \mathbb{P})$ be a complete probability space and for $T>0$ fixed let $g=(g(t))_{t \in[0, T]}$ be a Gaussian process on $(\Omega, \mathcal{F}, \mathbb{P})$ with covariance function

$$
R(t, s):=\int_{0}^{s} \int_{0}^{t} \phi(u, v) d u d v ; \quad t, s \in[0, T]
$$

where $\phi$ satisfies the following condition:

$\left(C_{R}\right) \phi \in L^{1}\left([0, T]^{2}, d s d t ; \mathbb{R}\right), \phi$ is symmetric, positive definite such that there exist $p \in(1, \infty)$ and $C \in(0, \infty)$ such that

$$
\int_{0}^{T} \int_{0}^{T}|f(u) f(v) \phi(u, v)| d u d v \leq C\|f\|_{L^{p}([0 ; T] ; \mathbb{R})}^{2} \quad \text { for all } f \in L^{p}([0, T] ; \mathbb{R}) .
$$


Remark 2.1. One can show that if $\phi(u, v)=\Psi(u-v), u, v \in[0, T]$ for some $\Psi \in L^{r}([0, T] ; \mathbb{R})$ with $r \in(1, \infty)$. Then the inequality in condition $\left(C_{R}\right)$ holds with $p:=\frac{2 r}{2 r-1}$. In particular, $\left(C_{R}\right)$ holds for $f B M$ with Hurst parameter $H \in\left(\frac{1}{2}, 1\right)$ where $\Psi(u)=H(2 H-1)|u|^{2 H-2}$ (see [12, Lemma 2.0.2 and Example 2.0.3]).

Under Condition $\left(C_{R}\right)$ for any separable real Hilbert space $\left(\mathcal{H},\langle,\rangle_{\mathcal{H}}\right)$ and $f \in L^{p}([0, T] ; \mathcal{H})$ in the usual way one can define a stochastic integral $\operatorname{Int}(f)(t):=\int_{0}^{t} f d g \in L^{2}(\Omega ; \mathcal{H}), t \in$ $[0, T]$, such that for all $f, h \in L^{p}([0, T] ; \mathcal{H})$

$$
\mathbb{E}\left\langle\int_{0}^{T} f(s) d g(s), \int_{0}^{T} h(s) d g(s)\right\rangle_{\mathcal{H}}=\int_{0}^{T} \int_{0}^{T}\left\langle f(s), h\left(s^{\prime}\right)\right\rangle_{\mathcal{H}} \phi\left(s, s^{\prime}\right) d s d s^{\prime} .
$$

$\operatorname{Int}(f)$ has $\mathbb{P}$-a.s. continuous sample paths in $\mathcal{H}$, provided $f \in L^{p+\epsilon}([0, T] ; \mathcal{H})$ for some $\epsilon>0$ (see [12, Propositions 2.4 and 2.8]).

Now let us pass to an infinite dimensional Gaussian process $G$ (replacing $g$ ) taking values in a separable Hilbert space $\left(U,\langle,\rangle_{U}\right)$ by taking independent copies $g_{n}, n \in \mathbb{N}$, of $\mathrm{g}$ on a complete probability space $(\Omega, \mathcal{F}, \mathbb{P})$, fixing an orthonormal basis $e_{n}, n \in \mathbb{N}$, of $\mathrm{U}$ and defining

$$
G(t):=\sum_{n=1}^{\infty} \sqrt{\lambda_{n}} g_{n}(t) e_{n}, t \in[0, T],
$$

where $\lambda_{n}>0$ are such that

$$
\sum_{n=1}^{\infty} \sqrt{\lambda_{n}}<\infty
$$

We note that as usual when dealing with $g_{n}$ with covariance given by (2.1) (i.e. $g_{n}$ is not a Browniem motion) one needs the stronger condition (2.5) rather than just assuming

$$
\sum_{n=1}^{\infty} \lambda_{n}<\infty
$$

Then for $h \in L^{p}([0, T] ; L(U, \mathcal{H}))$, where $L(U, \mathcal{H})$ denotes the set of all bounded linear operators from $U$ to $\mathcal{H}$, we can define

$$
\int_{0}^{t} h(s) d G(s):=\sum_{n=1}^{\infty} \sqrt{\lambda_{n}} \int_{0}^{t} h(s) e_{j} d g_{j}(s), \quad t \in[0, T],
$$

and one proves (cf. [12, Lemmas 2.0.10, 2.0.11 and Corollary 2.0.13]) 
Proposition 2.2. $\int_{0}^{\cdot} h(s) d G(s)$ is an $\mathcal{H}$-valued Gaussian process, which, if $h \in L^{p+\epsilon}([0, T] ; L(U, \mathcal{H}))$ for some $\epsilon>0$, has $\mathbb{P}$-a.s. continuous sample paths. Furthermore, for all $x, y \in \mathcal{H} ; h_{1}, h_{2} \in L^{p}([0, T] ; L(U, \mathcal{H}))$

$$
\begin{aligned}
& \mathbb{E}\left(\left\langle\int_{0}^{T} h_{1}(s) d G(s), x\right\rangle_{\mathcal{H}}\left\langle\int_{0}^{T} h_{2}(s) d G(s), y\right\rangle_{\mathcal{H}}\right) \\
= & \int_{0}^{T} \int_{0}^{T}\left\langle h_{2}\left(s^{\prime}\right) Q h_{1}(s)^{*} x, y\right\rangle_{\mathcal{H}} \phi\left(s, s^{\prime}\right) d s d s^{\prime}
\end{aligned}
$$

where $Q z:=\sum_{n=1}^{\infty} \lambda_{n}\left\langle z, e_{n}\right\rangle_{U} e_{n}, z \in U$, is the covariance operator of $G$. Finally,

$$
\begin{aligned}
& \mathbb{E}\left\langle\int_{0}^{T} h_{1}(s) d G(s), \quad \int_{0}^{T} h_{2}(s) d G(s)\right\rangle_{\mathcal{H}} \\
= & \int_{0}^{T} \int_{0}^{T} \operatorname{Tr}\left(h_{2}\left(s^{\prime}\right) Q h_{1}(s)^{*}\right) \phi\left(s, s^{\prime}\right) d s d s^{\prime} .
\end{aligned}
$$

\section{Main result}

Let $\left(H,<,>_{H}\right)$ be a separable real Hilbert space with dual $H^{*}$ and $V$ a reflexive Banach space such that $V \subset H$ continuously and densely. Then for its dual $V^{*}$ we have

$$
V \subset H \subset V^{*}
$$

continuously and densely, i.e. $\left(V, H, V^{*}\right)$ forms a Gelfand triple. We note that then $V^{*}$ and hence $V$ are also separable. Let $\left(U,<,>_{U}\right)$ be another separable real Hilbert space and $(W(t))_{t \in[0, T]}$ a cylindrical Wiener process on $U$ on some stochastic basis $\left(\Omega, \mathcal{F},\left(\mathcal{F}_{t}\right)_{t \in[0, T]}, \mathbb{P}\right)$. Let $G$ be defined as in Section 2 on $(\Omega, \mathcal{F}, \mathbb{P})$ (in particular, $C_{R}$ is assumed to hold) and suppose that $G$ is $\left(\mathcal{F}_{t}\right)$-adapted.

Now let us fix the conditions on the coefficients $A, B, h$ in (1.1). Let $L_{2}(U, H)$ denote the set of all Hilbert-Schmidt operators from $U$ to $H$ and let

$$
A:[0, T] \times V \times \Omega \rightarrow V^{*}, B:[0, T] \times V \times \Omega \rightarrow L_{2}(U, H)
$$

be progressively measurable, i.e. for every $t \in[0, T]$, these maps restricted to $[0, t] \times V \times \Omega$ are $\mathcal{B}([0, t]) \otimes \mathcal{B}(V) \otimes \mathcal{F}_{t}$-measurable. As usual by writing $A(t, v)$ we mean the map $\omega \mapsto A(t, v, \omega)$. Analogously for $B(t, v)$. We impose the following conditions on $A$ and $B$ :

(H1) (Hemicontinuity) For all $u, v, x \in V, \omega \in \Omega$ and $t \in[0, T]$ the map

$$
\mathbb{R} \ni \lambda \mapsto{ }_{V^{*}}\langle A(t, u+\lambda v, \omega), x\rangle_{V}
$$

is continuous. 
(H2) (Weak monotonicity) There exists $c \in \mathbb{R}$ such that for all $u, v \in V$

$$
\begin{aligned}
& 2_{V^{*}}\langle A(\cdot, u)-A(\cdot, v), u-v\rangle_{V}+\|B(\cdot, u)-B(\cdot, v)\|_{L_{2}(U, H)}^{2} \\
& \leq c\|u-v\|_{H}^{2} \text { on }[0, T] \times \Omega
\end{aligned}
$$

(H3) (Coercivity) There exist $\alpha \in(1, \infty), c_{1} \in \mathbb{R}, c_{2} \in(0, \infty)$ and an $\left(\mathcal{F}_{t}\right)$-adapted process $f \in L^{1}([0, T] \times \Omega, d t \otimes P)$ such that for all $v \in V, t \in[0, T]$

$$
2_{V^{*}}\langle A(t, v), v\rangle_{V}+\|B(t, v)\|_{L_{2}(U, H)}^{2} \leq c_{1}\|v\|_{H}^{2}-c_{2}\|v\|_{V}^{\alpha}+f(t) \quad \text { on } \Omega .
$$

(H4) (Boundedness) There exist $c_{3} \in[0, \infty)$ and an $\left(\mathcal{F}_{t}\right)$-adapted process $g \in L^{\frac{\alpha}{\alpha-1}}([0, T] \times$ $\Omega, d t \otimes P)$ such that for all $v \in V, t \in[0, T]$

$$
\|A(t, v)\|_{V^{*}} \leq g(t)+c_{3}\|v\|_{V}^{\alpha-1} \text { on } \Omega
$$

where $\alpha$ is as in (H3).

(H5) $h \in L^{p+\epsilon}([0, T] ; L(U, \mathcal{H}))$ for some $\epsilon>0$, where $\mathcal{H}:=V$ if $V$ is a Hilbert space or $\mathcal{H}:=H$ if $h=P$. $h$ for some orthogonal projection in $H$ with finite dimensional range in $V$.

Definition 3.1. A continuous $H$-valued $\left(\mathcal{F}_{t}\right)$-adapted process $X=(X(t))_{t \in[0, T]}$ is called a (variational) solution to (1.1), if

(i) $\hat{X} \in L^{\alpha}([0, T] \times \Omega, d t \times \mathbb{P} ; V) \cap L^{2}([0, T] \times \Omega, d t \times \mathbb{P} ; H)$

where $\alpha$ is as in (H3) and $\hat{X}$ is a $d t \times \mathbb{P}$-equivalence class of $X$ and $\mathbb{P}$-a.s.

(ii) $X(t)=X(0)+\int_{0}^{t} A(s, \bar{X}(s)) d s+\int_{0}^{t} B(s, \bar{X}(s)) d W(s)+\int_{0}^{t} h(s) d G(s)$,

where $\bar{X}$ is any $V$-valued progressively measurable $d t \otimes \mathbb{P}$-version of $\hat{X}$.

Now we claim and prove the main result:

Theorem 3.2. Under the above setting, for any given $X_{0} \in L^{2}\left(\Omega, \mathcal{F}_{0}, P, H\right)$, there exists a unique (variational) solution to (1.1)

Proof (sketch). Let $w(t), t \in[0, T]$, denote (the continuous version of $\int_{0}^{\cdot} h(s) d G(s)$ and define

$$
\bar{A}:[0, T] \times V \times \Omega \rightarrow V^{*}, \bar{B}:[0, T] \times V \times \Omega \rightarrow L_{2}(U, H)
$$

by 


$$
\begin{aligned}
& \bar{A}(t, v, \omega):=A(t, v+w(t)(\omega), \omega) \\
& \bar{B}(t, v, \omega):=B(t, v+w(t)(\omega), \omega)
\end{aligned}
$$

Then $\bar{A}, \bar{B}$ are again progressively measurable (see [12, Lemma 3.4]) and, since $w(t), t \in$ $[0, T]$, has strong moments of all orders as a Gaussian process, one can check that $\bar{A}, \bar{B}$ also satisfy (H1)-(H4) (cf. [12, Section 3]). Furthermore, $X$ is a solution to (1.1) if and only if

$$
Y(t):=X(t)-w(t), t \in[0, T]
$$

is a solution to

$$
d Y(t)=\bar{A}(t, Y(t)) d t+\bar{B}(t, Y(t)) d W(t) .
$$

But since $\bar{A}, \bar{B}$ satisfy (H1)-(H4), due to a general theorem on (variational) solutions to SPDE (see e.g. [10, Theorem 4.2.4] and the original paper [6]) there exists a unique (variational) solution to (3.2) (see [12, Section 3] for details).

Remark 3.3. It is possible to derive explicit bounds on the second strong moment of the solution in Theorem 2 (see [12, Section 4]).

Example 3.4. Since $A$ and $B$ in (1.1) satisfy the standard conditions (H1)-(H4) there are plenty of examples known from classical results in PDE (cf. e.g. [10, Subsection 4.1]) and more recent results on SPDE (see e.g. [1]). We refer to Section 5 in [12] where a number of them are worked out. We only mention here that nonlinear operators $A$ of the following type are included:

$$
\begin{aligned}
& A(u)=\triangle \Psi(u) \\
& A(u)=\operatorname{div} \beta(\nabla u),
\end{aligned}
$$

with mappings $\Psi: \mathbb{R} \rightarrow \mathbb{R}, \beta: \mathbb{R}^{d} \rightarrow \mathbb{R}^{d}$ respectively, which are continuous, polynomially bounded, monotone and coercive.

\section{References}

[1] J. Ren, M. Röckner, Y. Wang Stochastic generalized porous media and fast diffusion equations, J. Differential Equations 238 (2007), no. 1, 118-152.

[2] T.E. Duncan, B. Maslowski, B. Pasik-Duncan, Semilinear Stochastic Equations in a Hilbert Space with a Fractional Brownian Motion, Proc. of 23rd IFIP TC 7 Conf. on System Modelling and Optimization (2007), 104-106. 
[3] T.E. Duncan, B. Maslowski, B. Pasik-Duncan, Fractional Brownian Motion and Linear Stochastic Equations in Hilbert Space, Stoch. Dyn. 2 (2002), no. 2, 225-250.

[4] T.E. Duncan, B. Maslowski, B. Pasik-Duncan, Stochastic equations in Hilbert space with a multiplicative fractional Gaussian noise, Stochastic Processes and their Applications, 115(2005), 1357-1383.

[5] W. Grecksch, V.V. Anh, A Parabolic Stochastic Differential Equation With Fractional Brownian Motion Input, Stochastics and Probability Letters, 41(1999), 337-346.

[6] N.V. Krylov, B.L. Rozovskii, Stochastic Evolution Equations, Vol. 14, pp. 71-147, 256, Akad. Nauk SSSR, Vsesoyuz. Inst. Nauchn. i Tekhn. Informatsii, Moscow, 1979.

[7] B. Maslowski, D. Nualart, Evolution equations driven by a fractional Brownian Motion, J. Funct. Anal. 202 (2003), no. 1, 277-305.

[8] D. Nualart, Stochastic Calculus with respect to Fractional Brownian Motion, Annales de la Faculte des Sciences de Toulouse, Vol. 14, 2006, pp. 63-77.

[9] D. Nualart, P.A. Vuillermot, Variational solutions for partial differential equations driven by a fractional noise, Journal of Functional Analysis, 232(2006), 390-454.

[10] C. Prévôt, M. Röckner, A Concise Course on Stochastic Partial Differential Equations Lecture Notes in Mathematics, vol. 1905, Springer, 2007.

[11] S. Tindel, C. A. Tudor, F. Viens, Stochastic evolution equations with fractional Brownian motion, Probab. Theory Relat. Fields 127(2003), no. 2, 186.204.

[12] Y. Wang, Variational solutions to SPDE perturbed by a general Gaussian noise, PhDThesis, Purdue University 2009. 